ACCEPTED MANUSCRIPT

\title{
Strong-coupling charge density wave in monolayer $\mathrm{TiSe}_{2}$
}

To cite this article before publication: Matthew D Watson et al 2020 2D Mater. in press https://doi.org/10.1088/2053-1583/abafec

\section{Manuscript version: Accepted Manuscript}

Accepted Manuscript is "the version of the article accepted for publication including all changes made as a result of the peer review process, and which may also include the addition to the article by IOP Publishing of a header, an article ID, a cover sheet and/or an 'Accepted Manuscript' watermark, but excluding any other editing, typesetting or other changes made by IOP Publishing and/or its licensors"

This Accepted Manuscript is (c) 2020 IOP Publishing Ltd.

During the embargo period (the 12 month period from the publication of the Version of Record of this article), the Accepted Manuscript is fully protected by copyright and cannot be reused or reposted elsewhere.

As the Version of Record of this article is going to be / has been published on a subscription basis, this Accepted Manuscript is available for reuse under a CC BY-NC-ND 3.0 licence after the 12 month embargo period.

After the embargo period, everyone is permitted to use copy and redistribute this article for non-commercial purposes only, provided that they adhere to all the terms of the licence https://creativecommons.org/licences/by-nc-nd/3.0

Although reasonable endeavours have been taken to obtain all necessary permissions from third parties to include their copyrighted content within this article, their full citation and copyright line may not be present in this Accepted Manuscript version. Before using any content from this article, please refer to the Version of Record on IOPscience once published for full citation and copyright details, as permissions will likely be required. All third party content is fully copyright protected, unless specifically stated otherwise in the figure caption in the Version of Record.

View the article online for updates and enhancements. 
The transition metal dichalcogenides (TMDCs) are wellknown for hosting charge density waves (CDW) in their threedimensional bulk form, but the evolution of these instabilities in the two-dimensional monolayer limit remains poorly understood. In some cases, such as $2 \mathrm{H}-\mathrm{NbSe}_{2}$, the CDW ordering of a monolayer takes a similar form to the bulk material [1], albeit with reports of a dramatically enhanced transition temperature [2]. In other cases, however (notably $1 \mathrm{~T}^{-}-\mathrm{VSe}_{2}$ and $\mathrm{VTe}_{2}$ [3-6]), the monolayer ordering can be very different. Furthermore, polymorphs distinct from that of the bulk crystal structure can be stabilised in the monolayer, such as $1 \mathrm{~T}-\mathrm{NbSe}_{2}$ [7], which themselves can host unique instabilities. Given the further possibilities of exploring strain, stoichiometry/ variation, substrate interactions, gated devices and heterostructures, monolayer TMDCs offer an exciting new platform for exploring many-body instabilities in solids, offering the advantages of simplicity and tunability compared with their bulk counterparts.

One of the most well-known and intriguing TMDCs hosting CDW order is $\mathrm{TiSe}_{2}$. This compound is a narrow-gap semiconductor, which nonetheless undergoes an unusual charge ordering in bulk form at $T_{c}=200 \mathrm{~K}$, resulting in a $2 a \times 2 a \times 2 c$ lattice reconstruction [8]. The three-dimensional character of the CDW is linked to the finite $k_{z}$-dispersions of both the holeand electron-like states [9]. However, a similar $2 a \times 2 a$ charge instability is known to occur in the 2D limit of monolayer $\mathrm{TiSe}_{2}$ [10-16]. In particular, angle-resolved photoemission spectroscopy (ARPES) measurements on monolayer samples have shown prominent temperature-dependent signatures of the CDW [11-13], but the nature and origin of the instability remains controversial. Multiple scenarios have been proposed [17], with particular interest in the prospect of exciton condensate formation $[18,19]$. The problem continues to attract significant theoretical attention, with the monolayer environ- ment offering new opportunities for quantitative investigation of different theoretical approaches [20,21].

In this paper, we perform temperature-dependent ARPES measurements on monolayer $\mathrm{TiSe}_{2}$ samples grown by molecular beam epitaxy (MBE) on graphene-terminated SiC substrates. The system exhibits a narrow band gap of $80 \pm 20 \mathrm{meV}$ at room temperature, which opens up below $T_{c}$, reaching $180 \mathrm{meV}$ at low temperatures. We show how the instability has a strong-coupling nature, and highlight a contrasting behaviour of different atomic orbitals in the charge ordering instability. We find that the low temperature dispersions can be well described by a tight-binding model including the extra $d-p$ hybridisations introduced by the lattice displacements in the ordered phase, and identify the observed conduction band as a non-bonding state, associated with the $d_{3 z^{2}-r^{2}}$ orbital projection. Finally, via control of the sample stoichiometry, we demonstrate how the CDW ordering can be suppressed by introducing sufficient Se vacancies, establishing a critical doping level of 0.06(2) electrons per Ti.

\section{SYNTHESIS AND CHARACTERISATION}

Our 1T-TiSe ${ }_{2}$ samples are grown by van der Waals epitaxy on bilayer graphene-terminated $\mathrm{SiC}$ wafers (Fig. 1a) in a MBE system dedicated to chalcogenide growth (see Methods). Bilayer formation is avoided by using calibrated growth setpoints and the growth is stopped while the graphene streaks are still visible in the reflection high-energy electron diffraction (RHEED) images (e.g. Fig. 1(b)), leading to partial coverage of monolayer-height islands. The samples were grown at relatively low substrate temperatures to minimize Se vacancy formation $\left(300-400^{\circ} \mathrm{C}\right.$ ) and with slow growth rates (approx. $1 \mathrm{ML} / 75$ minutes) to optimise adatom diffusion during 
a
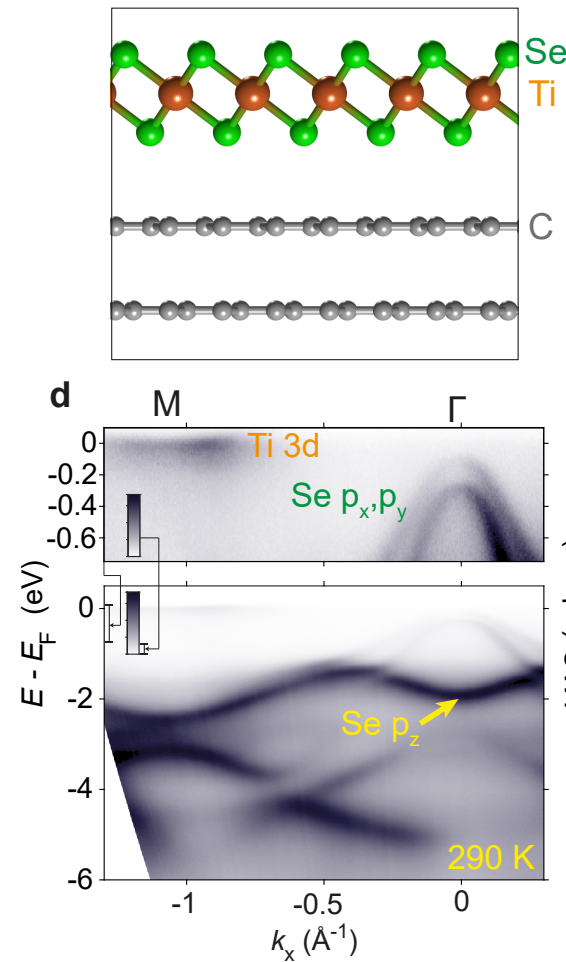

b
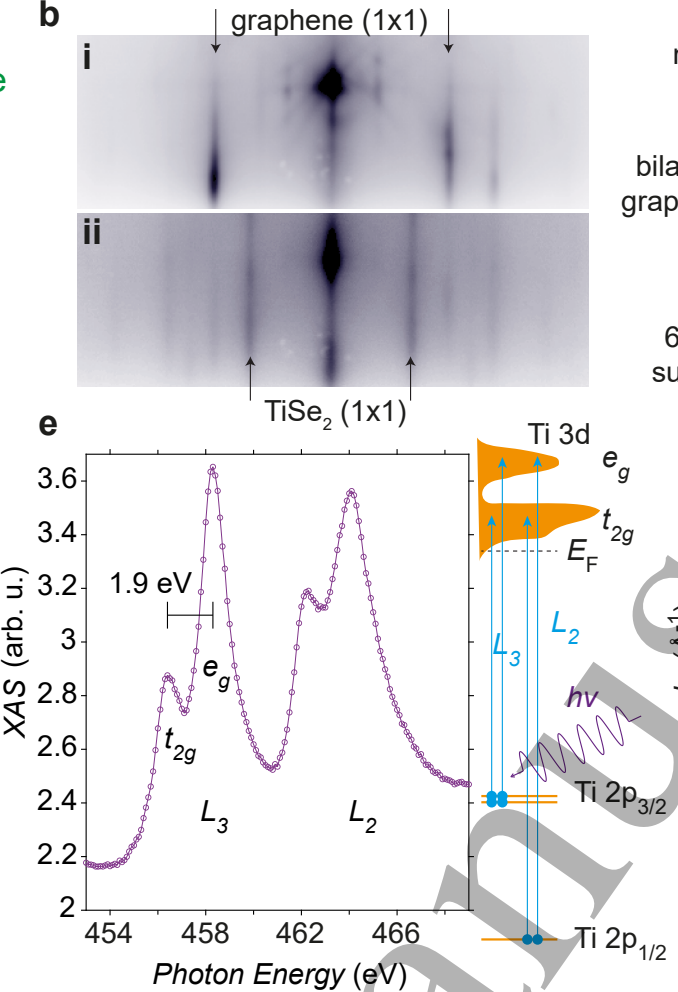

C

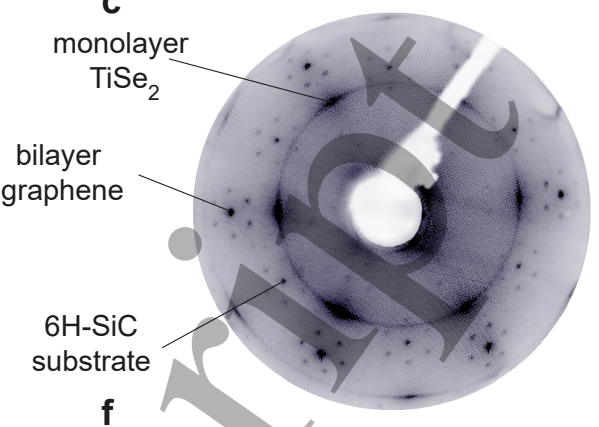

FIG. 1. a) Schematic structure, with a single layer of $1 \mathrm{~T}-\mathrm{TiSe}_{2}$ grown on bilayer graphene formed on the surface of a SiC substrate (not shown). b) RHEED images of i) graphene substrate before growth and ii) after growth of monolayer $\mathrm{TiSe}_{2}$. c) LEED pattern (incident beam energy of $170 \mathrm{eV}, T=20 \mathrm{~K}$ ). d) Overview of the electronic structure, dominated by Se-derived valence bands. The upper panel displays the same data as the lower panel, but with different limits on the colourscale. e) XAS measurement; the structure of the absorption edges reflects the crystal field splitting of the unoccupied Ti $3 d$ states as shown schematically. $L_{2,3}$ refers to the standard notation for transitions from the two spin-orbit split Ti $2 p$ states. f) Fermi surface measurement at $T=10 \mathrm{~K}$; hexagon is $1 \times 1$ Brillouin zone, see also Fig 2(f).

the growth [22].

For van der Waals epitaxy, there is only a weak interaction between the epilayer and substrate. As a result, negligible strain is coupled into the epilayer: the in-plane lattice constant as determined from our RHEED and low-energy electron diffraction (LEED) images (Fig. 1(b,c)) are consistent with the bulk values. The lack of strong interaction with the substrate gives rise to a degree of disorder in the rotational alignment between the monolayer and the substrate. This is evident in the LEED image in Fig. 1(c). Both the $\mathrm{SiC}$ and graphene give rise to sharp spots with visible superstructure peaks. For the monolayer $\mathrm{TiSe}_{2}$, however, while the diffraction is still peaked along the direction aligned with the underlying graphene, the Bragg spots are azimuthally-broadened (typical full-width half-maximum of $\sim 10-15^{\circ}$ ), leading to a weak ring of continuous intensity visible at all azimuthal angles. The presence of rotational misalignment has an impact on the ARPES measurements that follow; for example the low-temperature Fermi surface in Fig. 1(f) shows a similar azimuthal distribution of intensity to the LEED data (as also evident in literature measurements of samples prepared in a similar manner [16]). However, the rotational disorder does not critically affect the band dispersions as measured along the important $\Gamma-\mathrm{M}$ orientation, as this high-symmetry radial cut is less sensitive to the azimuthal smearing effect. Moreover, this configuration, with weak interaction with the substrate, allows for investigation of the intrinsic electronic properties of the monolayer system in a "quasi-freestanding" environment.

\section{NORMAL-STATE ELECTRONIC STRUCTURE}

We first examine the gross features of the normal-state electronic structure of monolayer $\mathrm{TiSe}_{2}$. As shown in Fig. 1(d), we find a bandwidth of approximately $5.5 \mathrm{eV}$ containing six valence bands, primarily deriving from the $4 p$ orbitals on the two Se sites. At the $\Gamma$ point, a prominent band at $-2 \mathrm{eV}$ can be attributed to a Se $p_{z}$ state; in fact, most of the brightest features in Fig. 1(d) have $p_{z}$ character, which is related to a strong matrix element in this particular geometry and photon energy. The out-of-plane $p_{z}$-derived states bear the strongest imprint of the spatial confinement of the monolayer sample. In the bulk, pronounced interlayer hopping processes for the $p_{z}$ orbitals lead to a large dispersion in the out-of-plane $\Gamma$-A direction for the $p_{z}$-derived states, with the energy of the $p_{z^{-}}$derived band comparable with the other hole bands at the $\Gamma$ point [9]. However, in monolayer form, quantum confinement 

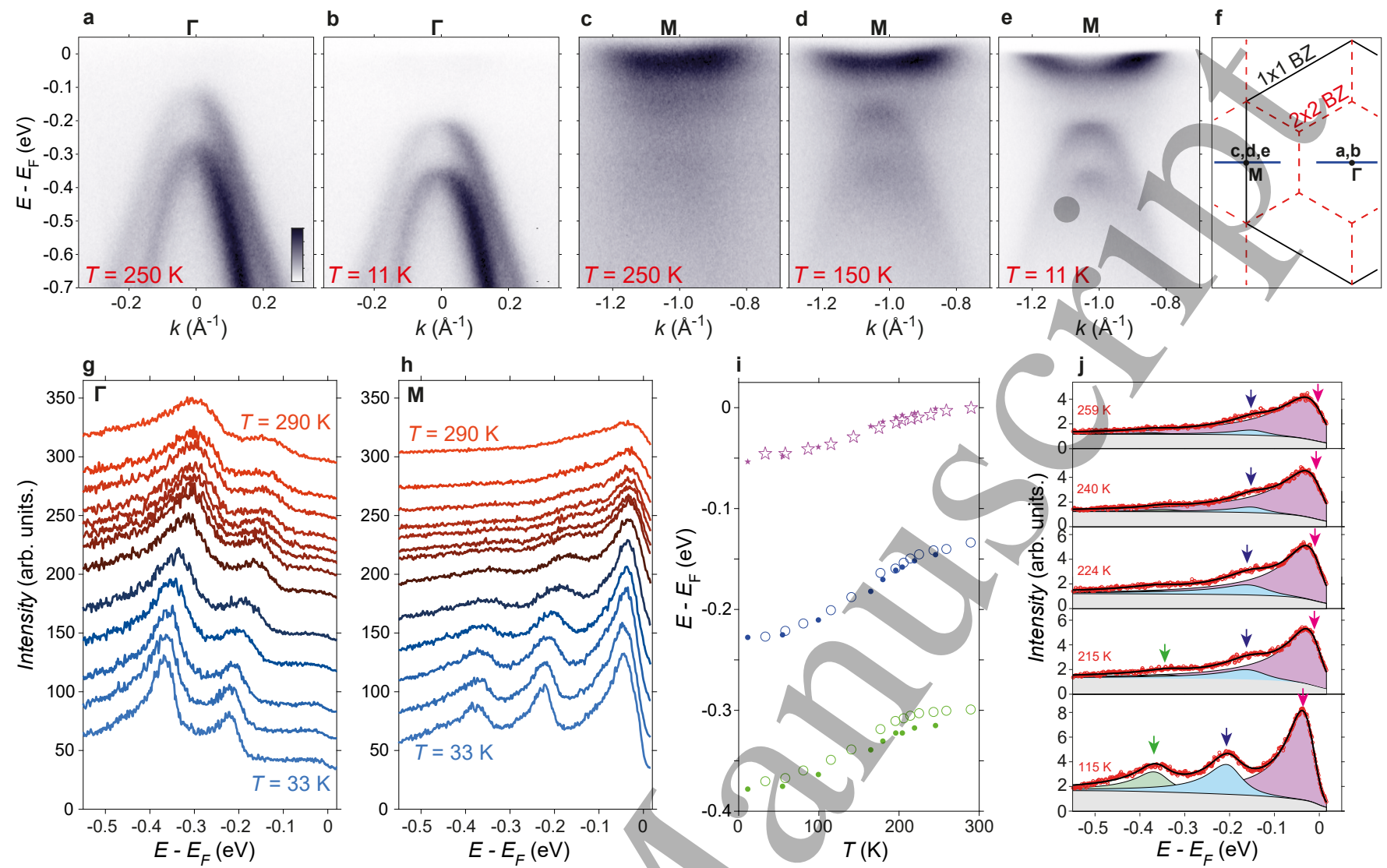

FIG. 2. a,b) Measurements of the hole pockets at a) $250 \mathrm{~K}$ - above $T_{c}$ and b) $11 \mathrm{~K}$ - well below $T_{c}$. c-e) Temperature-dependent measurements at the $\mathrm{M}$ point (temperatures indicated on the figure), showing an electron pocket as well as the development of backfolded hole pockets. $\mathrm{f}$ ) Brillouin zone corresponding to the high-temperature $(1 \times 1)$ and low-temperature $(2 \times 2)$ structure, with the location of the measurements in (a-e) indicated. g,h) Temperature-dependent EDCs extracted for the $\Gamma$ and $\mathrm{M}$ points respectively (measured from a different sample to that shown in (a-e)). i) Extracted temperature-dependence of EDC peak positions, from a fitting analysis of the EDCs at $\Gamma$ (valence bands, open circles) and $\mathrm{M}$ (conduction band, open stars). The smaller filled symbols are from equivalent analysis for the sample shown in (a-e). $\mathrm{j}$ ) Selected EDCs at the M point, and multi-peak fit. Peak positions are noted with arrows; note that the conduction band position is quite affected by the Fermi-Dirac function at high temperatures. The blue peak can be resolved even up to $T=259 \mathrm{~K}$, indicating the persistence of some backfolded spectral weight at temperatures above $T_{c}$.

pushes the $p_{z}$ states to higher binding energies, as observed here. The pair of hole bands that approach, but do not reach, the chemical potential at $\Gamma$ are assigned as $p_{x, y}$ character.

At the M point in Fig. 1(d), spectral weight from the bottom of an electron-like band is observed. However, only a very small fraction of this band is populated, as the Ti $3 d$ states from which it derives should be generally unoccupied. These states may be detected via X-ray Absorption Spectroscopy (XAS, Fig. 1(e)) which probes dipole-allowed transitions between the Ti $2 p$ core levels and unoccupied Ti $3 d$ states, as shown by the schematic diagram in Fig. 1(e). This data allows us to identify separately the transitions into the unoccupied $t_{2 g}$ and $e_{g}$ states [23]. The resolution of distinct $t_{2 g}$ and $e_{g}$ manifolds, separated by $1.9 \mathrm{eV}$, indicates that the $t_{2 g}$ bandwidth is smaller than the octahedral crystal field splitting, and points to a much lower bandwidth for the unoccupied $\mathrm{Ti} 3 d$ states than for the occupied Se $4 p$ states. This is expected from the more local nature of the Ti $3 d$ orbitals, and is consistent with the much flatter parabolic dispersion of the occupied part of the conduction band at $\mathrm{M}$ evident in our ARPES measurements, as compared with the Se $4 p$ hole bands at $\Gamma$ (Fig. 1(d)). The partial occupation of the conduction band is due to a slight off-stoichiometry of the samples, and potentially also due to a small charge transfer from the substrate; all our monolayer samples were found to be $n$-doped in this manner. We will demonstrate control of this doping level below (Fig. 4), and explore the consequences of its variation on the CDW.

\section{EXPERIMENTAL SIGNATURES OF A STRONG-COUPLING CDW}

With the global electronic structure established, we now concentrate on the bands within a few hundred $\mathrm{meV}$ of $E_{F}$, which are relevant for the CDW physics. The $p_{z}$-derived band lies outwith this range due to the confinement effects discussed above, and therefore cannot participate in the CDW 
ordering. In Fig. 2 we show temperature-dependent ARPES measurements across the CDW transition. Similar data has been already reported [11, 12, 14-16], but here we focus in more detail on the implications of such measurements regarding the nature of the instability.

The Se $4 p_{x, y}$-derived hole-like states at the $\Gamma$ point are shown in Fig. 2(a). Without spin-orbit coupling, these hole bands would be degenerate at the $\Gamma$ point, but the sizeable spin-orbit interaction for these states splits the bands by $\approx$ $200 \mathrm{meV}$ [21]. Neither hole band crosses the chemical potential, and both remain below the M-point conduction band minimum (Fig. 2(c)). There has been some contention over whether bulk $\mathrm{TiSe}_{2}$ is a semimetal or semiconductor in the high-temperature phase $[9,17,24]$, but monolayer $\mathrm{TiSe}_{2}$ is undoubtedly a narrow-gap semiconductor at $300 \mathrm{~K}$; we estimate the normal state band gap to be $80 \pm 20 \mathrm{meV}$ (see Supplementary Fig. S1). Thus, the 'Fermi surface' in the high temperature phase consists only of rather small electron pockets due to the unintentional doping of the $\mathrm{Ti} 3 d$ conduction band. This condition is far removed from the typical picture of weak-coupling CDWs as an instability of a large Fermi surface with significant nesting.

Upon cooling the sample, the hole bands shift to substantially higher binding energy (Fig. 2(b)), as can also be seen from the temperature-dependent EDCs at the $\Gamma$ point in Fig. 2(g). The shape of the band also changes, developing a more flattened dispersion near the valence band maximum. These are both signatures of a hybridisation process occurring at low temperatures. The temperature-dependent binding energy of both valence bands exhibit kinks at $\approx 220 \mathrm{~K}$, which we assign as the CDW critical temperature, $T_{c}$. It is important to note that the two hole bands couple to the CDW as a pair; even though the lower hole band maximum is at $\approx-300 \mathrm{meV}$, it experiences a similar band shift to the upper branch. The fact that a band at such a high binding energy is contributing significantly to the hybridisation is evidence that the energy scale of the hybridisation interaction, $\Delta$, must be large - on the order of hundreds of meV. This implies that $2 \Delta / k_{B} T_{c} \gg 3.53$, far exceeding the value for weak-coupling electron-phonon superconductors. We investigate this quantitatively below, but already this qualitative finding implies that the CDW transition in monolayer $\mathrm{TiSe}_{2}$ is in the strongcoupling regime.

Consistent with this, we find a strong backfolded spectral weight of the valence bands from the $\Gamma$ to the $M$ points below $T_{c}$ (Fig. 2(d,e)). Such a band backfolding is a famous signature of the CDW ordering in $\mathrm{TiSe}_{2}[11-13,17,18,25,26]$. It is allowed as, for a $2 \times 2$ reconstruction, the $M$ point becomes equivalent to the $\Gamma^{*}$ point of the reduced Brillouin zone (Fig. 2(f)). The intensity that we find in Fig. 2(d,e) is, however, remarkably strong and can be detected down to at least $0.7 \mathrm{eV}$ below $E_{F}$. The spectral weight of backfolded features is typically associated with the magnitude of $\Delta$ [17], which again points to a large energy scale of the hybridisation.

Moreover, we find that signatures of the backfolded spectral weight at $\mathrm{M}$ persist above $T_{c}$. This is apparent in energy dis- tribution curves (EDCs) from the M point, shown in Fig. 2(j), where an extra shoulder feature is discernible in the spectrum even at $259 \mathrm{~K}$, well above $T_{c}$. Upon cooling, this feature gains spectral weight and develops into the upper backfolded valence band $T_{c}$ [27]. Its presence above $T_{c}$ points to precursor fluctuations of the order parameter, sufficient to backfold spectral weight on the fast timescale of photoemission, but without the development of static long-range order. Such precursor fluctuations are another hallmark of a/strong-coupling second-order phase transition [17].

\section{SELECTIVE HYBRIDISATIONS}

Despite these clear signatures of a strong hybridisation process being in play, surprisingly the occupied portion of the conduction band is not pushed away, and there remains an electron pocket in the ground state. In fact, the bottom of the conduction band actually shifts continuously to higher binding energy upon cooling (Fig. 2(i)), which we attribute to a substantial, but essentially trivial chemical potential effect $[28,29]$. There is no anomaly in the band position at $T_{c}$, and no evident backfolding of the conduction band spectral weight to the zone centre in the low-temperature state, both pointing to a lack of band hybridisation of this state. These unusual characteristics raise an important and hitherto unconsidered question for monolayer $\mathrm{TiSe}_{2}$ : if the observed electron pocket has a non-bonding characteristic, then with which states did the hole pockets hybridise?

To come to a better understanding of the hybridisation process, which ultimately provides the energy gain required for the CDW to occur, we turn to tight-binding modelling. A recent theoretical study introduced a suitable model [20], in which the parameters of the model were constrained by fitting to DFT band dispersions. Here, we make two adaptations; firstly, we adjust the energy difference of the Ti- $d$ and Se$p$ states in order to match the experimental band gap in the normal state, and secondly we introduce an on-site spin-orbit coupling term on the Se sites - see Methods for more details.

In Fig. 3(a), we overlay ARPES data obtained at $300 \mathrm{~K}$ with the bands of the tight-binding model, showing excellent agreement. At low temperatures, we implement the CDW ordering by considering a $2 \times 2$ supercell, rendering the $M$ and $\Gamma$ points equivalent in reciprocal space (Fig. 2(f)). For simplicity we only consider the displacements of the $\mathrm{Ti}$ atoms, and rescale the $p-d$ hopping parameters according to the change in SeTi bond lengths (making use of the calculated values for the derivatives of the hopping parameters in Ref. [20]). This creates a new part of the Hamiltonian which breaks the symmetry of the original $1 \times 1$ unit cell, creating terms that hybridise the two hole pockets and three backfolded electron pockets (one from each M-point). For the hybridised plot in Fig. 3(b), we fix the Ti displacements at $0.02 a$, and also allow for a different chemical potential as is observed experimentally [28].

Three backfolded electron pockets, one from each of the M-points, would be degenerate at $\Gamma^{*}$ for infinitesimal atomic 
displacements. The tight-binding analysis reveals how these backfolded bands split and develop distinctly different behaviour as a result of the new orbital hybridisations allowed by the finite atomic displacements in the ordered phase. In Fig. 3(c), we see that a pair of hybridised states pick up an increased Se character and are shifted upwards in energy. These are the states which have hybridised with the hole pockets, causing them to flatten and to be pushed above $E_{\mathrm{F}}$. Without spin-orbit coupling, these would form a doublet, but due to their acquired partial Se character the spin-orbit coupling lifts their degeneracy. These hybridised bands lie a little above $E_{F}$ in the unoccupied states. They are thus inaccessible to ARPES, however they should be accessible indirectly via optical transitions that become allowed below $E_{F}$, similar to signatures measured on bulk $\mathrm{TiSe}_{2}[30,31]$, or more directly by inverse photoemission spectroscopy or in tunneling measurements.

There remains a singlet state below $E_{F}$ in Fig. 3(c), however, which does not develop any increased Se character. This indicates that it is not hybridised with the valence band states, and thus remains below $E_{\mathrm{F}}$. The fact that this state does not bond can be better understood by the projection onto the $\mathrm{Ti}$ states in Fig. 3(d), where it is evident that the non-bonding state corresponds to the Ti $d_{3 z^{2}-r^{2}}$ projection [9] [32]. The $d_{3 z^{2}-r^{2}}$ projection is equiyalent to a fully symmetric combination of the wavefunctions of the three backfolded conduction bands, creating a highly-symmetric wavefunction which is forbidden to hybridise with any combination of $p_{x}$ and $p_{y}$ orbitals (at least, exactly at the $\Gamma^{*}$ point of the reconstructed cell).

The dichotomous behaviour of the bonding and nonbonding electron band branches reconciles the unusual experimental signatures discussed above. Since the observed electron pocket is from the non-bonding band, it has no coupling to the new periodicity of the CDW, and thus does not appear backfolded at the $\Gamma$ point in the experimental measurements (i.e., it has negligible spectral weight). Moreover, its energy does not change as a function of hybridisation in the model; its shift in Fig. 3(b) compared to (a) is only due to an overall chemical potential shift. This explains the lack of any apparent anomaly in the temperature-dependence of the observed conduction band on cooling through $T_{c}$ (Fig. 2(h)).

Importantly, this means that the experimental band gap is not an adequate representation of the energy scale of the CDW. As indicated in Fig. 3(b) the band gap $E_{G}$ measures only the hybridised valence bands with respect to the nonbonding conduction band, not to the hybridised states above $E_{F}$. Thus, while the band gap does open up below $T_{c}$ (Supplementary Fig. S1), considering $E_{G}$ alone would give a significant underestimate of the true energy scale of $2 \Delta$. The full hybridisation scale can instead be estimated from the energy gap between the uppermost valence band and the lowest hybridised branch of the conduction band, $E_{G}^{\prime}$ in Fig. 3(b), which we relate to the true interaction scale $\Delta$ using:

$$
E_{G}^{\prime}=\sqrt{E_{G 0}^{2}+(2 \Delta)^{2}} .
$$

Taking $E_{G 0}=80 \mathrm{meV}$ (Supplementary Fig. S1)) and $E_{G}^{\prime}=218 \mathrm{meV}$ (from our optimised tight binding analysis, Fig. 3(b)) yields $2 \Delta=203 \mathrm{meV}$. Taking $T_{c}=220 \mathrm{~K}$ from the kink in the valence band energies in Fig. 2(i), the ratio $2 \Delta / k_{B} T$ is found to be 10.7 . This places the CDW instability of monolayer $\mathrm{TiSe}_{2}$ firmly in the strong-coupling limit [17].

Proper identification of the relevant hybridised states is essential for understanding the energy scale that underpins the charge ordering instability. This, in turn, can give important insights into its microscopic origin. There has been a long-standing discussion of whether electronic/excitonic interactions are an important, or even crucial, factor in enabling the structural distortion to occur in $\mathrm{TiSe}_{2}[18,19,25-$ $27,31,33]$. Indeed, it is sometimes argued that hybridisation energy scales on the order of those observed here would 
be too large to explain the CDW instability from structural terms alone, arguably pointing to an important role of additional electronic interactions [20] and an excitonic insulator scenario [19]. However it has been and remains challenging to tackle this issue quantitatively, since the structural and putative electronic terms break the same symmetry and are argued to be cooperative [20].

The simplifications of the band structure in the monolayer give a fresh chance for quantitative analysis, by comparison with tight-binding modelling in a markedly simplified system as compared to the bulk [9]. Within our model, the magnitude of the band hybridisation is set purely by the Ti atomic displacements; i.e., we do not invoke any other electronic terms. Importantly, we find good agreement on the energies of the hybridised bands when setting the value of the Ti displacement to $0.02 a$; this optimal value is in very good agreement with the experimentally-determined lattice distortion in monolayer $\mathrm{TiSe}_{2}$ [34], which is similar to the bulk value [8]. In other words, within our model, the new symmetry-breaking single-particle hopping terms in the Hamiltonian induced by the known atomic displacements can alone account for the full energy scale of the hybridisation found experimentally. Our results would thus be consistent with a transition driven dominantly by electron-phonon coupling, which is enabled by the resulting electronic energy gain from band hybridisation in the vicinity of the Fermi level which becomes allowed due to the atomic displacements, similar to a regular CDW. We caution, however, that the single-particle spectral function as obtained from ARPES can only ever offer indirect evidence for or against the presence of two-particle bound states such as excitons, and our measurements here do not exclude a cooperative role for excitonic interactions in mediating the transition [35]. Nonetheless, our results highlight that the structurally-induced hybridisations undoubtedly play a strong role in enabling this phase transition to occur.

\section{SUPPRESSING THE CDW BY ELECTRON DOPING}

While our measurements indicate that stoichiometric $\mathrm{TiSe}_{2}$ should be a narrow-gap semiconductor, all MBE-grown samples that we fabricated were found to be degenerately $n$ doped. This may partially arise due to an intrinsic charge transfer from the substrate. However, another known effect is that Se has a very high vapour pressure, making it likely for Se vacancies to form. This suggests possibilities to tune the carrier density via deliberate introduction of Se vacancies. Upon heating the film in vacuum to $\sim 500{ }^{\circ} \mathrm{C}$, a film that originally had a band structure resembling Fig. 4(a) was transformed into a highly electron-doped system, with a very large electron pocket as shown in Fig. 4(c), presumably due to the release of Se atoms. We found, however, that more controllable sample stoichiometry can be achieved by tuning the the growth temperature in conjunction with post-growth annealing steps. Both the sample growth and post-growth annealing are performed in a substantial partial pressure of $\mathrm{Se}$ $\left(\approx 1 \times 10^{-7}\right.$ mbar $)$, during which there is a dynamical balance between Se vacancies being formed and refilled, depending on parameters including the substrate temperature. In Fig. 4(b,e) we show the ARPES results on a film after growth at a relatively high temperature of $650^{\circ} \mathrm{C}$ and annealing at $500^{\circ} \mathrm{C}$, resulting in a moderately doped (i.e. Se deficient) sample where the electron pocket has expanded to a $k_{F}$ of $0.27 \AA^{-1}\left(n_{e} \approx\right.$ $0.10(1)$ ). Interestingly, there is no evidence for backfolding of the hole pockets in this sample, and the hole pockets are found at higher energy in Fig. 4(e) as compared/with Fig. 4(d), indicating the absence of hybridisation here. These signatures demonstrate that the CDW becomes unfavourable when the filling of the electron pocket becomes too large.

We show in Fig. 4(f) the relationship between the intensity of the backfolded hole pockets, a proxy for the strength of the CDW order, and the $k_{F}$ value of the electron pocket, which we use as a proxy for the electron doping, as determined across 9 separate samples. As the electron band becomes increasingly filled, the backfolded intensity gradually reduces until it becomes completely suppressed, indicating the destruction of the CDW order. By extrapolation, we find a critical value of around $k_{F} \approx 0.22(2) \AA$, which approximately corresponds to $0.06(2)$ extra electrons per Ti [36]. The critical doping level for suppression of the CDW in monolayer $\mathrm{TiSe}_{2}$ is thus comparable with the bulk material $[37,38]$.

\section{DISCUSSION AND CONCLUSIONS}

On the one hand, we can consider that the CDW of $\mathrm{TiSe}_{2}$ is rather robust; it not only exists in both bulk and monolayer forms, but also in all known few-layer forms (e.g. Ref. [13, 39]). Moreover, the CDW persists in monolayers grown on different substrates; here we have considered only $\mathrm{SiC} /$ graphene, but similar data including sharp signatures of the backfolded bands can be obtained on graphite (HOPG, see Supplementary Fig. S3), while CDW signatures have also been reported for $\mathrm{TiSe}_{2}$ grown on $\mathrm{MoS}_{2}$ [15]. The CDW also seems to be relatively insensitive to the higher levels of disorder present in MBE-grown films compared to high-quality single crystals. Alongside the numerous spectral signatures discussed above, these observations point towards a strongcoupling nature of the charge ordering.

On the other hand, we have shown that the charge ordering in monolayer $\mathrm{TiSe}_{2}$ can be suppressed by a modest electron doping of only $0.06(2)$ extra electrons per Ti. The suppression of a charge-ordered state by a tuning parameter such as electron-doping is commonly observed across many families of quantum materials [40-42]. For weak-coupling instabilities, one might argue that the suppression is linked to changes in the Fermi surface that move the system away from a nesting condition, or away from a peak in the density of states at the Fermi level. However, given that $\mathrm{TiSe}_{2}$ is a narrow-gap semiconductor, and the evidence for a strong-coupling-type instability here, a different rationale is necessary. From the excitonic insulator perspective, it could be reasoned that the 


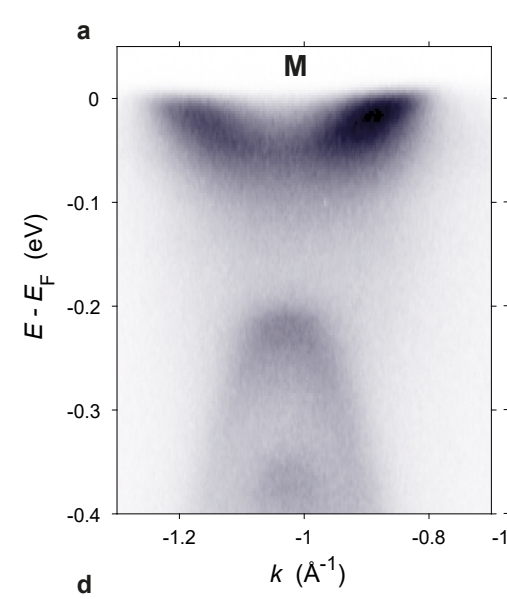

b
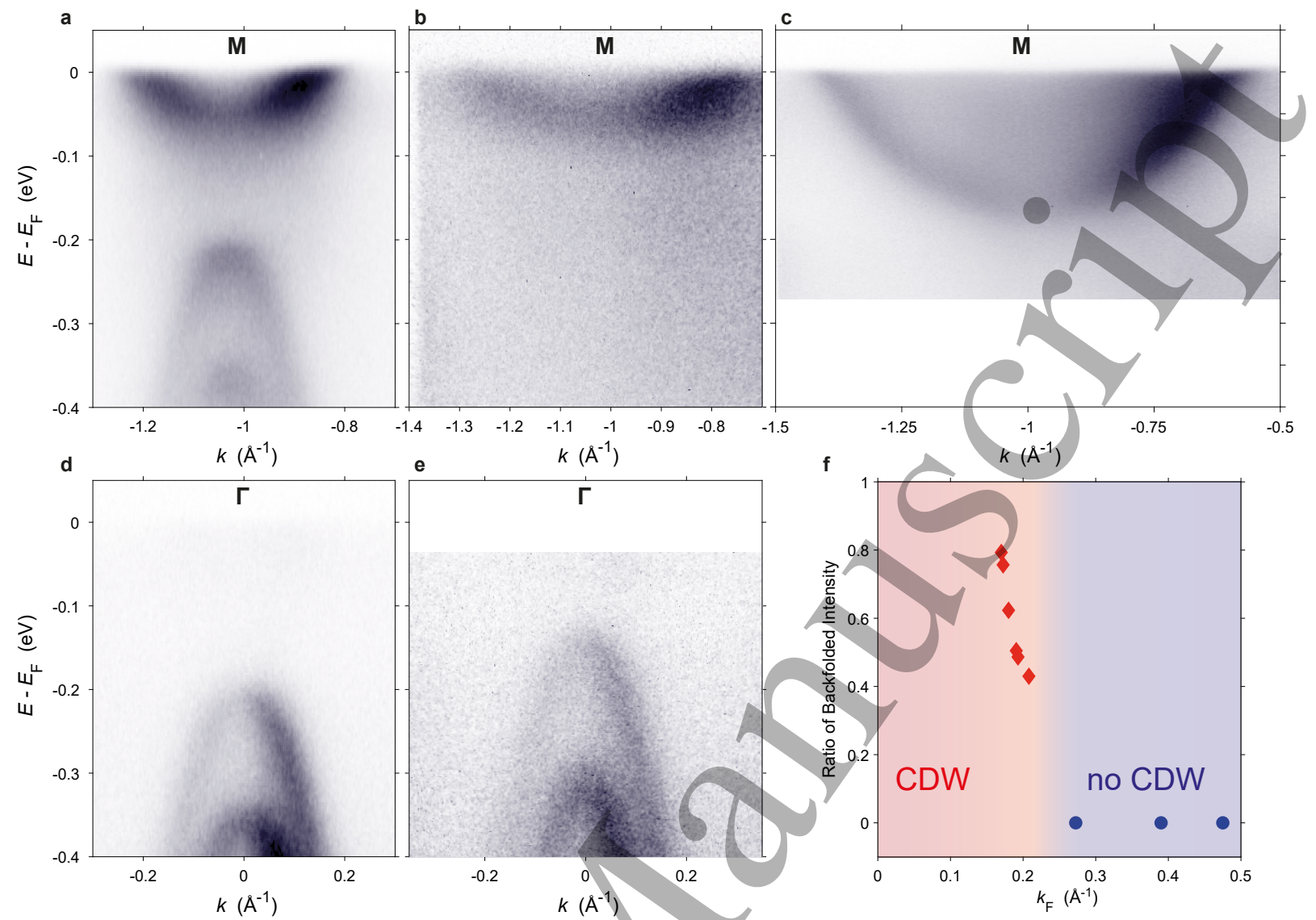

FIG. 4. Variation of ARPES spectra with sample preparation method. a) Sample grown at $300{ }^{\circ} \mathrm{C}$ and annealed at $500{ }^{\circ} \mathrm{C}$ under a Se flux after growth; b) Sample grown at $650{ }^{\circ} \mathrm{C}$ with a $500^{\circ} \mathrm{C}$ post-growth anneal in Se flux; c) Sample grown at $350{ }^{\circ} \mathrm{C}$ and annealed in vacuum at $\sim 500^{\circ} \mathrm{C}$ following growth. $\mathrm{d}, \mathrm{e}$ ) Hole band dispersions at $\Gamma$ corresponding to samples (a) and (b), respectively. f) Comparison of the backfolded intensity across multiple samples. Where backfolding was observed, it became weaker in intensity with increasing $k_{F}$, while the sample shown in $(b, e)$ marks the first point where no backfolding was observed. The metric used for determining the magnitude of backfolding is defined in Supplemental Figure S2.

added free carriers tend to screen out the Coulomb interaction between electrons and holes, i.e. doping suppresses the ordering by suppressing the microscopic interaction. However, in such a case, the weaker screening environment of the monolayer as compared to the bulk might be expected to strengthen the instability, leading to a higher $T_{c}$, while the $T_{c}$ observed here is only slightly higher (by $\approx 20 \mathrm{~K}$ ) than in the bulk, again pointing to a strong importance of the lattice instability here.

Irrespective of the microscopics of the interaction, our tight binding analysis gives a rationalisation of the doping dependence from a purely energetic perspective. The downward energetic shift of the occupied hole bands due to the electronhole hybridisation is the primary source of the energy gain in the CDW phase [25]. However, partial filling of the electron bands means that these also contribute to the overall energetic considerations. We recall that the conduction band minimum in the ordered state is of non-bonding character (Fig. 3), and it does not participate in the CDW. The additional filling of this non-bonding band alone therefore does not give rise to a significant energetic penalty (compared with the equivalent filling of the electron band in the undistorted phase) and the CDW remains stable as the energy gain in the hybridised hole bands is unaffected. However, if the doping level becomes sufficiently high that the hybridised branches of the conduction band states, which are located just above $E_{F}$ in Fig. 3(bd), would become occupied, then the overall electronic energy gain of the CDW ordering would become quickly nullified. Thus, up to a point, electron doping will not significantly weaken the CDW order (e.g. even our lowest-doping samples have a doping level of $\sim 0.03$ per Ti). Once a doping threshold is reached, however, the hybridised branch of the conduction band would become sufficiently populated so as to remove the energetic gain of forming the $2 \times 2$ phase; the CDW transition thus becomes suppressed. In our tight-binding model, occupation of the hybridised electron states would commence when the $k_{F}$ of the non-bonding conduction band (in the ordered phase) exceeds $0.18 \AA^{-1}$, which compares well with the experimental finding (Fig. 4(f)) that no signatures of CDW order are found in samples with $k_{F} \gtrsim 0.21 \AA^{-1}$.

In conclusion, we have demonstrated a strong-coupling 
CDW instability in monolayer $\mathrm{TiSe}_{2}$ that hybridises electron and hole-like states across the indirect band gap. However, the conduction band that is observed at low temperatures has peculiar properties that indicate non-bonding characteristics, as confirmed by our tight-binding modelling. With control of the growth temperature and post-growth annealing, the stoichiometry of the samples may be controlled, principally by the introduction of Se vacancies. Although the CDW is initially tolerant to some off-stoichiometry, with increased filling of the conduction band beyond $x \sim 0.06(2)$ we found that the CDW may be completely suppressed. Monolayer $\mathrm{TiSe}_{2}$ is thus shown to be a simpler, and more tunable, system than bulk $\mathrm{TiSe}_{2}$, and as such provides an excellent test-case for quantitative analysis of the much-debated electronic and structural instability. Moreover, in bulk $\mathrm{TiSe}_{2}$, a small superconducting dome is found near the critical point for suppression of the CDW order [37]; given the similarity in dopingdependence of the monolayer system observed here to the bulk, our results suggest an exciting possibility that such a superconducting dome could be realised also for monolayer $\mathrm{TiSe}_{2}$, motivating future studies of this intriguing CDW material in monolayer form.

\section{ACKNOWLEDGMENTS}

We thank Chiara Bigi, Lewis Hart, Kai Rossnagel, Andreas Rost, and Peter Wahl for useful discussions. We gratefully acknowledge funding from The Royal Society, The Leverhulme Trust, and the European Research Council (through the ERC714193-QUESTDO project). The MBE growth facility was funded through the Engineering and Physical Sciences Research Council (EPSRC) under Grant No. EP/M023958/1. $\mathrm{KU}$ and OJC acknowledge the EPSRC for PhD studentship support via Grant Nos. EP/L015110/1 and EP/K503162/1, and IM the International Max Planck Research School for Chemistry and Physics of Quantum Materials. We thank SOLEIL synchrotron for access to the CASSIOPEE beamline (proposal 20171202) and Elettra synchrotron for access to the APE-HE beamline, which both contributed to the results presented here. This work has been partially performed in the framework of the Nanoscience Foundry and Fine Analysis (NFFA-MIUR Italy Progetti Internazionali) facility. The research leading to this result has been supported by the project CALIPSOplus under Grant Agreement 730872 from the EU Framework Programme for Research and Innovation HORIZON 2020.

\section{METHODS}

Sample preparation: $7 \times 3 \mathrm{~mm}^{2}$ wafers of $n$-type $6 \mathrm{H}$ $\mathrm{SiC}(0001)$ were used for graphene synthesis. After cleaning using acetone and isopropanol, two of these wafers were made into a sandwich with the Si terminated surfaces facing each other with $0.1 \mathrm{~mm}$ Ta spacers between them on either end.
The wafers are then loaded into a dedicated UHV chamber equipped with a pyrometer to measure the temperature. After annealing at $\sim 600{ }^{\circ} \mathrm{C}$ for two hours using direct current heating, the temperature is further increased to $1500^{\circ} \mathrm{C}$ for 7 minutes to create a bilayer of graphene. Once loaded into the growth chamber, these substrates are degassed at a temperature at least $100{ }^{\circ} \mathrm{C}$ higher than the growth temperature for $\sim 40$ minutes.

The base pressure of the growth chamber is $\sim 3 \times 10^{-10}$ mbar. A valyed cracker cell with $5 \mathrm{~N}$-pure $\mathrm{Se}$ and a high temperature cell with $4 \mathrm{~N}$-pure $\mathrm{Ti}$ are used as sources. Materials fluxes are measured in beam equivalent pressure (BEP) using a retractable ion gauge. A variety of growth temperatures and parameters were used, but a typical sample (GM2 - 156) was grown at $400{ }^{\circ} \mathrm{C}$ for 70 minutes, using a Ti cell temperature of $1340{ }^{\circ} \mathrm{C}$ and Se cell temperature of $140{ }^{\circ} \mathrm{C}$ while maintaining the Se cracker zone at $500{ }^{\circ} \mathrm{C}$. Cell temperatures were adjusted for other sample growths in order to maintain a constant Ti BEP of $\sim 6 \times 10^{-10}$ mbar and at least an order of magnitude greater Se flux. A post-growth annealing, under Se partial pressure, was found to improve the data quality on some samples. A more wide-ranging analysis of the growth modes achieved in different parameter regimes can be found in Ref. [22].

ARPES: ARPES measurements in Figures 1, 2 and 4 were performed using a helium lamp source, i.e. a photon energy $h \nu=21.2 \mathrm{eV}$. The photoelectrons are analysed with a Specs Phoibos 225 analyser. Reference measurements were made on polycrystalline gold samples to establish the Fermi level. The vacuum level was typically $3 \times 10^{-11}$ mbar. The ARPES system is connected by UHV transfers to the growth chamber. ARPES measurements in Figure 3 were obtained at beamline CASSIOPEE, at the synchrotron Soleil using a photon energy of $40 \mathrm{eV}$. In additional, x-ray absorption spectroscopy measurements (Fig. 1(e)) were performed in total electron yield mode at the APE Beamline of Elettra synchrotron [43]. For both of these experiments, the samples were prepared with an amorphous Se cap which was then removed by heating to $\sim 220{ }^{\circ} \mathrm{C}$ at the beamline.

Tight-binding model: Our model is an adaptation of that provided by Kaneko et al [20]. We keep all of the values of the hopping parameters from that study, but adjust the onsite energy of the $p$ orbitals from -2.171 to $-2.456 \mathrm{eV}$; the lowering of the Se $p$ states relative to the Ti $d$ states is how we reach a semiconducting (rather than semimetallic) state in the undistorted phase, in agreement with our experiments. Our model has a band gap of $71 \mathrm{meV}$ (see Supplementary Fig. S1 for further discussion of the normal state band gap). We also add an on-site spin-orbit coupling term on the Se sites $(\lambda=0.105 \mathrm{eV})$. This interaction opens up the substantial splitting of the Se $p_{x}, p_{y}$ states at $\Gamma$. For best agreement with the data we add an overall chemical potential term of $0.205(0.17) \mathrm{eV}$ in the undistorted (distorted) phases. The distorted phase is implemented by constructing a $2 \times 2$ expanded unit cell, and 
rescaling the hopping parameters between Ti and neighbouring Se sites according to the known pattern of atomic displacements [8], only implementing the displacements of Ti for simplicity. For this we make use of the derivatives of the $p-d$ hopping parameters listed in Ref. [20]. For the calculations in Fig. 3(b-d), the Ti displacements have a magnitude of $0.02 * a$, where $a$ is the lattice constant.

* matthew.watson@diamond.ac.uk

† philip.king@st-andrews.ac.uk

[1] M. M. Ugeda, A. J. Bradley, Y. Zhang, S. Onishi, Y. Chen, W. Ruan, C. Ojeda-Aristizabal, H. Ryu, M. T. Edmonds, H.Z. Tsai, A. Riss, S.-K. Mo, D. Lee, A. Zettl, Z. Hussain, Z.X. Shen, and M. F. Crommie, Characterization of collective ground states in single-layer $\mathrm{NbSe}_{2}$, Nat. Phys. 12, 92 (2016).

[2] X. Xi, L. Zhao, Z. Wang, H. Berger, L. Forró, J. Shan, and K. F. Mak, Strongly enhanced charge-density-wave order in monolayer $\mathrm{NbSe}_{2}$, Nature Nanotechnology 10, 765 (2015).

[3] J. Feng, D. Biswas, A. Rajan, M. D. Watson, F. Mazzola, O. J. Clark, K. Underwood, I. Marković, M. McLaren, A. Hunter, D. M. Burn, L. B. Duffy, S. Barua, G. Balakrishnan, F. Bertran, P. Le Fèvre, T. K. Kim, G. van der Laan, T. Hesjedal, P. Wahl, and P. D. C. King, Electronic Structure and Enhanced ChargeDensity Wave Order of Monolayer VSe 2 , Nano Letters 18, 4493 (2018).

[4] P. Chen, W. W. Pai, Y.-H. Chan, V. Madhavan, M. Y. Chou, S.-K. Mo, A.-V. Fedorov, and T.-C. Chiang, Unique Gap Structure and Symmetry of the Charge Density Wave in Single-Layer $\mathrm{VSe}_{2}$, Phys. Rev. Lett. 121, 196402 (2018).

[5] G. Duvjir, B. K. Choi, I. Jang, S. Ulstrup, S. Kang, T. Thi Ly, S. Kim, Y. H. Choi, C. Jozwiak, A. Bostwick, E. Rotenberg, J.G. Park, R. Sankar, K.-S. Kim, J. Kim, and Y. J. Chang, Emergence of a Metal-Insulator Transition and High-Temperature Charge-Density Waves in $\mathrm{VSe}_{2}$ at the Monolayer Limit, Nano Letters 18, 5432 (2018).

[6] Y. Wang, J. Ren, J. Li, Y. Wang, H. Peng, P. Yu, W. Duan, and S. Zhou, Evidence of charge density wave with anisotropic gap in a monolayer $\mathrm{VTe}_{2}$ film, Phys. Rev. B 100, 241404 (2019).

[7] Y. Nakata, K. Sugawara, R. Shimizu, Y. Okada, P. Han, T. Hitosugi, K. Ueno, T. Sato, and T. Takahashi, Monolayer 1T-NbSe 2 as a Mott insulator, NPG Asia Materials 8, e321 (2016).

[8] F. J. Di Salvo, D. E. Moncton, and J. V. Waszczak, Electronic properties and superlattice formation in the semimetal $\mathrm{TiSe}_{2}$, Phys. Rev. B 14, 4321 (1976).

[9] M. D. Watson, O. J. Clark, F. Mazzola, I. Marković, V. Sunko, T. K. Kim, K. Rossnagel, and P. D. C. King, Orbital- and $k_{z^{-}}$ Selective Hybridization of Se $4 p$ and Ti $3 d$ States in the Charge Density Wave Phase of $\mathrm{TiSe}_{2}$, Phys. Rev. Lett. 122, 076404 (2019).

[10] J.-P. Peng, J.-Q. Guan, H.-M. Zhang, C.-L. Song, L. Wang, K. He, Q.-K. Xue, and X.-C. Ma, Molecular beam epitaxy growth and scanning tunneling microscopy study of $\mathrm{TiSe}_{2}$ ultrathin films, Phys. Rev. B 91, 121113 (2015).

[11] P. Chen, Y.-H. Chan, X.-Y. Fang, Y. Zhang, M. Y. Chou, S.-K. Mo, Z. Hussain, A.-V. Fedorov, and T.-C. Chiang, Charge density wave transition in single-layer titanium diselenide, Nature Communications 6, 8943 (2015).

[12] K. Sugawara, Y. Nakata, R. Shimizu, P. Han, T. Hitosugi, T. Sato, and T. Takahashi, Unconventional Charge-Density-
Wave Transition in Monolayer 1T-TiSe 2 , ACS Nano 10, 1341 (2016).

[13] P. Chen, Y.-H. Chan, M.-H. Wong, X.-Y. Fang, M. Y. Chou, S.K. Mo, Z. Hussain, A.-V. Fedorov, and T.-C. Chiang, Dimensional Effects on the Charge Density Waves in Ultrathin Films of $\mathrm{TiSe}_{2}$, Nano Letters 16, 6331 (2016).

[14] S. Kolekar, M. Bonilla, Y. Ma, H. C. Diaz, and M. Batzill, Layer- and substrate-dependent charge density wave criticality in $1 \mathrm{~T}-\mathrm{TiSe}_{2}, 2 \mathrm{D}$ Materials 5, 015006 (2017).

[15] S. Kolekar, M. Bonilla, H. C. Diaz, M. Hashimoto, D. Lu, and M. Batzill, Controlling the Charge Density Wave Transition in Monolayer TiSe $e_{2}$ : Substrate and Doping Effects, Advanced Quantum Technologies 1, 1800070 (2018).

[16] T. Jia, S. N. Rebec, S. Tang, K. Xu, H. M. Sohail, M. Hashimoto, D. Lu, R. G. Moore, and Z.-X. Shen, Epitaxial growth of $\mathrm{TiSe}_{2} / \mathrm{TiO}_{2}$ heterostructure, 2D Materials 6, 011008 (2018).

[17] K. Rossnagel, On the origin of charge-density waves in select layered transition-metal dichalcogenides, Journal of Physics: Condensed Matter 23, 213001 (2011).

[18] H. Cercellier, C. Monney, F. Clerc, C. Battaglia, L. Despont, M. G. Garnier, H. Beck, P. Aebi, L. Patthey, H. Berger, and L. Forró, Evidence for an Excitonic Insulator Phase in $1 T-\mathrm{TiSe}_{2}$, Phys. Rev. Lett. 99, 146403 (2007).

[19] A. Kogar, M. S. Rak, S. Vig, A. A. Husain, F. Flicker, Y. I. Joe, L. Venema, G. J. MacDougall, T. C. Chiang, E. Fradkin, J. van Wezel, and P. Abbamonte, Signatures of exciton condensation in a transition metal dichalcogenide, Science 358, 1314 (2017).

[20] T. Kaneko, Y. Ohta, and S. Yunoki, Exciton-phonon cooperative mechanism of the triple- $q$ charge-density-wave and antiferroelectric electron polarization in $\mathrm{TiSe}_{2}$, Phys. Rev. B 97, 155131 (2018).

[21] D. Pasquier and O. V. Yazyev, Excitonic effects in twodimensional $\mathrm{TiSe}_{2}$ from hybrid density functional theory, Phys. Rev. B 98, 235106 (2018).

[22] A. Rajan, K. Underwood, F. Mazzola, and P. D. C. King, Morphology control of epitaxial monolayer transition metal dichalcogenides, Phys. Rev. Materials 4, 014003 (2020).

[23] Here the symmetry language refers to approximate octahedral symmetry, which is not strictly applicable as the Ti site has only trigonal symmetry. However, the trigonal distortion is small, and the octahedral crystal field splitting remains the dominant energy scale.

[24] M.-L. Mottas, T. Jaouen, B. Hildebrand, M. Rumo, F. Vanini, E. Razzoli, E. Giannini, C. Barreteau, D. R. Bowler, C. Monney, H. Beck, and P. Aebi, Semimetal-to-semiconductor transition and charge-density-wave suppression in $1 T-\mathrm{TiSe}_{2-x} \mathrm{~S}_{x}$ single crystals, Phys. Rev. B 99, 155103 (2019).

[25] T. E. Kidd, T. Miller, M. Y. Chou, and T.-C. Chiang, ElectronHole Coupling and the Charge Density Wave Transition in $\mathrm{TiSe}_{2}$, Phys. Rev. Lett. 88, 226402 (2002).

[26] K. Rossnagel, L. Kipp, and M. Skibowski, Charge-densitywave phase transition in $1 T-\mathrm{TiSe}_{2}:$ Excitonic insulator versus band-type Jahn-Teller mechanism, Phys. Rev. B 65, 235101 (2002).

[27] C. Monney, E. F. Schwier, M. G. Garnier, N. Mariotti, C. Didiot, H. Beck, P. Aebi, H. Cercellier, J. Marcus, C. Battaglia, H. Berger, and A. N. Titov, Temperature-dependent photoemission on $1 T-\mathrm{TiSe}_{2}$ : Interpretation within the exciton condensate phase model, Phys. Rev. B 81, 155104 (2010).

[28] M. D. Watson, A. M. Beales, and P. D. C. King, On the origin of the anomalous peak in the resistivity of $\mathrm{TiSe}_{2}$, Phys. Rev. B 99, 195142 (2019).

[29] C. Monney, E. F. Schwier, M. G. Garnier, N. Mariotti, C. Did- 
iot, H. Cercellier, J. Marcus, H. Berger, A. N. Titov, H. Beck, and P. Aebi, Probing the exciton condensate phase in 1T$\mathrm{TiSe}_{2}$ with photoemission, New Journal of Physics 12, 125019 (2010).

[30] G. Li, W. Z. Hu, D. Qian, D. Hsieh, M. Z. Hasan, E. Morosan, R. J. Cava, and N. L. Wang, Semimetal-to-Semimetal Charge Density Wave Transition in $1 T-\mathrm{TiSe}_{2}$, Phys. Rev. Lett. 99, 027404 (2007).

[31] C. Monney, M. Puppin, C. W. Nicholson, M. Hoesch, R. T. Chapman, E. Springate, H. Berger, A. Magrez, C. Cacho, R. Ernstorfer, and M. Wolf, Revealing the role of electrons and phonons in the ultrafast recovery of charge density wave correlations in 1T-TiSe 2 , Phys. Rev. B 94, 165165 (2016).

[32] Here, we refer to the $d_{3 z^{2}-r^{2}}$ projection in the crystallographic basis, i.e. with $z$ out of the plane.

[33] C. Chen, B. Singh, H. Lin, and V. M. Pereira, Reproduction of the Charge Density Wave Phase Diagram in $1 T-\mathrm{TiSe}_{2}$ Exposes its Excitonic Character, Phys. Rev. Lett. 121, 226602 (2018).

[34] X.-Y. Fang, H. Hong, P. Chen, and T.-C. Chiang, X-ray study of the charge-density-wave transition in single-layer $\mathrm{TiSe}_{2}$, Phys. Rev. B 95, 201409 (2017).

[35] M. Porer, U. Leierseder, J.-M. Ménard, H. Dachraoui, L. Mouchliadis, I. E. Perakis, U. Heinzmann, J. Demsar, K. Rossnagel, and R. Huber, Non-thermal separation of electronic and structural orders in a persisting charge density wave, Nature Materials 13, 857 (2014).

[36] For the purposes of this calculation we assume that the minor axis of the elliptical electron pocket, which we do not directly measure, is 4 times shorter than the major axis.

[37] E. Morosan, H. W. Zandbergen, B. S. Dennis, J. W. G. Bos, Y. Onose, T. Klimczuk, A. P. Ramirez, N. P. Ong, and R. J.
Cava, Superconductivity in $\mathrm{Cu}_{x} \mathrm{TiSe}_{2}$, Nature Physics 2, 544 (2006).

[38] J. F. Zhao, H. W. Ou, G. Wu, B. P. Xie, Y. Zhang, D. W. Shen, J. Wei, L. X. Yang, J. K. Dong, M. Arita, H. Namatame, M. Taniguchi, X. H. Chen, and D. L. Feng, Evolution of the Electronic Structure of $1 T-\mathrm{Cu}_{x} \mathrm{TiSe}_{2}$, Phys. Rev. Lett. 99, 146401 (2007).

[39] P. Goli, J. Khan, D. Wickramaratne, R. K. Lake, and A. A. Balandin, Charge Density Waves in Exfoliated Films of van der Waals Materials: Evolution of Raman Spectrûm in TiSe2, Nano Letters 12, 5941 (2012).

[40] P. J. Hirschfeld, M. M. Korshunov, and I. I. Mazin, Gap symmetry and structure of fe-based superconductors, Reports on Progress in Physics 74, 124508 (2011).

[41] R. Ang, Y. Tanaka, E. Ieki, K. Nakayama, T. Sato, L. J. Li, W. J. Lu, Y. P. Sun, and T. Takahashi, Real-Space Coexistence of the Melted Mott State and Superconductivity in Fe-Substituted $1 T-\mathrm{TaS}_{2}$, Phys. Rev. Lett. 109, 176403 (2012).

[42] R. Ang, Y. Miyata, E. Ieki, K. Nakayama, T. Sato, Y. Liu, W. J. Lu, Y. P. Sun, and T. Takahashi, Superconductivity and bandwidth-controlled Mott metal-insulator transition in $1 T$ $\mathrm{TaS}_{2-x} \mathrm{Se}_{x}$, Phys. Rev. B 88, 115145 (2013).

[43] G. Panaccione, I. Vobornik, J. Fujii, D. Krizmancic, E. Annese, L. Giovanelli, F. Maccherozzi, F. Salvador, A. De Luisa, D. Benedetti, A. Gruden, P. Bertoch, F. Polack, D. Cocco, G. Sostero, B. Diviacco, M. Hochstrasser, U. Maier, D. Pescia, C. H. Back, T. Greber, J. Osterwalder, M. Galaktionov, M. Sancrotti, and G. Rossi, Advanced photoelectric effect experiment beamline at Elettra: A surface science laboratory coupled with Synchrotron Radiation, Review of Scientific Instruments 80, 043105 (2009).
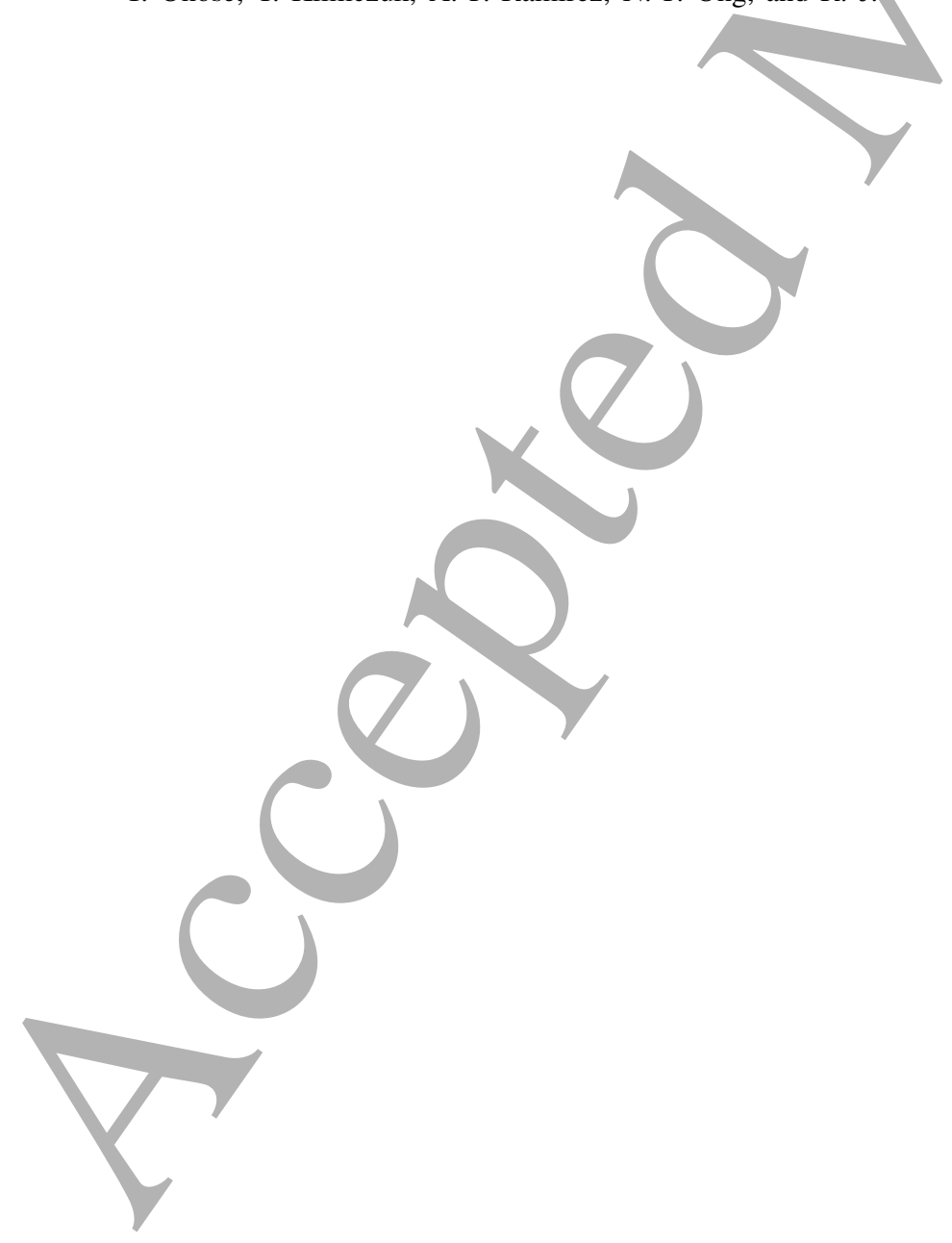